\title{
Control químico del ácaro rojo de las palmas Raoiella indica Hirst (Acari: Tenuipalpidae)
}

\section{Chemical control of the red palm mite Raoiella indica Hirst (Acari: Tenuipalpidae)}

\author{
Azalia Correa-Méndez ${ }^{1}$, Rodolfo Osorio-Osorio ${ }^{1 *}$, Luis Ulises Hernández-Hernández ${ }^{1}$, Efraín de la \\ Cruz-Lázaro $^{1}$, César Márquez-Quiroz ${ }^{1}$, Rosa Ma. Salinas-Hernández ${ }^{1}$ \\ ${ }^{1}$ Universidad Juárez Autónoma de Tabasco, División Académica de Ciencias Agropecuarias, Km 25 carretera \\ Villahermosa-Teapa. 86000. Centro, Tabasco, México \\ *Autor de correspondencia: rodolfo.osorio@ujat.mx
}

Nota científica recibida: 20 de enero de 2017 aceptada: 21 de septiembre de 2017

RESUMEN. El objetivo del trabajo fue comparar la efectividad biológica de 19 acaricidas comerciales para el control de poblaciones de Raoiella indica Hirst en Tabasco, México. Los acaricidas se asperjaron sobre el follaje de plantas de cocotero híbrido (Alto del Pacífico x Enano Malayo), previamente infestadas con $R$. indica, en dosis y frecuencia de aplicación recomendadas. Se registró el número de ácaros que permanecieron vivos en la superficie abaxial del follaje a los 2, 7, 14 y 21 d después de la aplicación. Los acaricidas bifentrina, acequinocyl, bifenazate, spiromesifen, amitraz, fenpyroximate, clorfenapir, azufre, clorhidrato de formetanato, milbemectina, propargite y fenazaquin fueron los más efectivos, al reducir las poblaciones de $R$. indica en más del $95 \%$ a los 7 d después de la aplicación. Se propone el uso secuenciado de estos acaricidas en la región, basándose en sus modos de acción, para un control químico racional de la plaga.

Palabras clave: Acaricidas, Cocos nucifera L., efectividad biológica, grupos toxicológicos

ABSTRACT. The aim of this study was to compare the biological effectiveness of 19 commercial acaricides for the control of Raoiella indica Hirst populations in Tabasco, Mexico. The acaricides were sprayed on the foliage of hybrid coconut plants (Alto del Pacifico x Enano Malayo), previously infested with $R$. indica, at the recommended dosage and frequency of application. The number of mites that remained alive on the abaxial surface of the foliage at 2, 7, 14 and $21 \mathrm{~d}$ after application was recorded. The acaricides bifenthrin, acequinocyl, bifenazate, spiromesifen, amitraz, fenpyroximate, chlorfenapyr, sulfur, formetanate hydrochloride, milbemectin, propargite and fenazaquin were the most effective, reducing the populations of $R$. indica by more than $95 \%$ at $7 \mathrm{~d}$ after application. The sequenced use of these acaricides in the region is proposed, based on their modes of action, for a rational chemical control of the pest.

Key words: Acaricides, Cocos nucifera L., biological effectiveness, toxicological groups

\section{INTRODUCCIÓN}

El ácaro rojo de las palmas Raoiella indica Hirst fue reportado por primera vez en el continente americano en el 2004, en la isla Martinica (Flechtmann y Etienne 2004). A pesar de las medidas cuarentenarias establecidas, se dispersó en las otras islas del Caribe y América Latina (Rodrigues et al. 2007, Kane et al. 2012). El ácaro causa daños en cultivos de importancia económica, como el cocotero (Cocos nucifera L.), bananos y plátanos (Musa spp.), palma de aceite (Elaeis guineensis Jacq.) y varias especies de palmas ornamentales y heliconias (Carrillo et al. 2012). En México se detectó por primera vez en el 2009 en los municipios de Benito Juárez e Isla Mujeres, Quintana Roo (NAPPO 2009), por lo que el Servicio Nacional de Sanidad, Inocuidad y Calidad Agroalimentaria, 
Correa-Méndez et al.

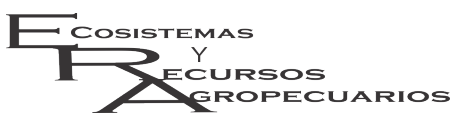

Control químico del ácaro rojo

Ecosist. Recur. Agropec.

5(14):319-326,2018

a partir de abril de 2010, implementó la campaña contra el ácaro rojo, cuyo objetivo fue evitar que la plaga ocasione daños de importancia económica en unidades de producción agrícola (SENASICA 2017). En el 2016, el gobierno federal aplicó un presupuesto de 19.06 millones de pesos para la campaña contra el ácaro rojo en los estados de Campeche, Chiapas, Colima, Guerrero, Jalisco, Michoacán, Nayarit, Oaxaca, Quintana Roo, Sinaloa, Tabasco, Veracruz y Yucatán. Esta campaña fitosanitaria se enfoca en la detección y manejo de brotes poblacionales de $R$. indica, mediante la aplicación periódica de acaricidas y podas sanitarias del material vegetativo infestado. Recomendando los acaricidas abamectina, spirodiclofen y azufre elemental, autorizados de forma temporal por la Comisión Federal para la Protección contra Riesgos Sanitarios (COFEPRIS) (SENASICA 2015).

El control químico de los ácaros es crucial en los programas de manejo integrado de plagas a nivel mundial y es poco probable que se elimine (Van Leeuwen et al. 2015); debido a que sirve para controlar brotes poblacionales (Fountain et al. 2010) o mitigar su dispersión (De Assis et al. 2013). Los ácaros incluyen especies con altas tasas de reproducción y ciclos de vida cortos (Van Leeuwen et al. 2010), lo que incrementa su potencial para desarrollar resistencia a los acaricidas (Whalon et al. 2008). La recomendación para manejar la resistencia contempla acciones como alternancia, secuenciación, rotación y uso de mezclas de compuestos con mecanismos de acción diferentes (Marcic 2012). Por lo que el desafío principal que enfrenta el control químico efectivo es reconocer la efectividad biológica de los acaricidas bajo condiciones locales para diversificar el mecanismo de mortalidad de la plaga (Whalon et al. 2008). Debido a que el uso de un ingrediente activo único para el control de plagas propicia la resistencia acelerada (Whalon et al. 2008). Por lo anterior el objetivo del presente trabajo fue comparar la efectividad biológica de 19 acaricidas para el control de $R$. indica en plantas de cocotero híbrido (Alto del Pacífico x Enano Malayo) en Tabasco, México.

\section{MATERIALES Y MÉTODOS}

\section{Sitio experimental}

El experimento se realizó en la ranchería Chipilinar $2^{a}$ Sección, municipio de Jalapa, Tabasco $\left(17^{\circ} 47.614^{\prime}\right.$ LN y $92^{\circ} 47.764^{\prime}$ LO) durante la época de secas que abarca de marzo a mayo, que se caracteriza por temperaturas altas y lluvias escasas (Larios-Romero y Hernández 1987). Estas condiciones ambientales favorecen el incremento poblacional de $R$. indica en sus plantas hospederas (Polanco-Arjona et al. 2017).

\section{Infestación del material vegetal}

Se adquirieron 80 plantas de cocotero híbrido (Alto del Pacífico $x$ Enano Malayo) de 18 meses de edad, en el vivero Hacienda Campo Real del municipio de Comalcalco, Tabasco. Las plantas se trasladaron al sitio experimental, donde por seis meses se les proporcionó riegos y fertilización química a dosis de $50 \mathrm{~g}$ de superfosfato triple por planta. La infestación experimental de $R$. indica en cada planta, se realizó de forma dirigida a los foliolos de la parte media de cuatro frondas, localizadas en la parte media del estrato arbóreo. En cada planta, se realizaron cuatro infestaciones a intervalos de dos semanas, del 16 de marzo al 27 de abril de 2016. En cada infestación, se colocaron 10 secciones de foliolo infestados por colonias de $R$. indica, sujetándolos a la superficie abaxial de los foliolos a infestar con clips, para permitir el establecimiento y colonización de los ácaros. La longitud de cada sección foliar infestante varió de 5 a $10 \mathrm{~cm}$, dependiendo de la densidad de colonias de ácaros, con la finalidad de uniformizar la cantidad de ácaros colocados por fronda. El material biológico se obtuvo de plantas de cocotero híbrido infestadas de forma natural por $R$. indica y $\sin$ antecedentes de tratamiento químico, localizadas en las inmediaciones del sitio experimental.

\section{Diseño experimental}

El experimento se estructuró bajo un diseño de bloques completos al azar con cuatro repeticiones. La unidad experimental consistió de una 
planta de cocotero infestada previamente por $R$. indica. Un día antes de la aplicación de los tratamientos, se realizó el muestreo del número de ácaros adultos de $R$. indica por foliolo por planta, tomando una muestra aleatoria de cuatro foliolos por planta, localizados en la parte media de las frondas del estrato arbóreo medio. De acuerdo con Roda et al. (2012), este tipo de muestreo aleatorio estratificado proporciona buena estimación de la densidad poblacional de $R$. indica en plantas de cocotero. El conteo de ácaros se realizó con una lupa de 20X dirigida a la superficie abaxial de los foliolos seleccionados. Estos foliolos se etiquetaron para evaluaciones subsecuentes después de la aplicación de los tratamientos. De acuerdo con el número promedio de ácaros adultos encontrados por foliolo por planta, se formaron cuatro bloques de 20 plantas: el primer bloque de 69 a 113 ácaros adultos, el segundo de 124 a 162, el tercero de 179 a 239 y el cuarto de 254 a 739 .

\section{Evaluación de tratamientos}

Se evaluaron 19 tratamientos (Tabla 1) correspondientes a los acaricidas comerciales, los cuales se agrupan en 15 grupos toxicológicos diferentes de acuerdo con la clasificación del Comité de Acción de Resistencia a los Insecticidas (IRAC) (Sparks y Nauen 2015). Son productos químicos para uso agrícola y control de diferentes especies de ácaros fitófagos de frutales, hortalizas y ornamentales; todos con registro vigente en la Comisión Federal para la Protección contra Riesgos Sanitarios (COFEPRIS 2015). Las dosis y frecuencias de aplicación se manejaron de acuerdo con las instrucciones de uso del producto. La aplicación de los acaricidas se realizó por aspersión manual con un atomizador con capacidad de $2 \mathrm{~L}$ (Marca Flo Master $\left.{ }^{R}\right)$, con una presión de salida de $1.1 \mathrm{Kg} \mathrm{cm}^{2}$ y un gasto de $50 \mathrm{ml}$ de solución por planta. Para evitar la contaminación por deriva, cada planta se asperjó por separado dentro de una estructura de plástico de $3 \times 4 \times 4 \mathrm{~m}$ de alto, largo y ancho. Luego de la aplicación, las plantas se colocaron a distancia de $3 \times 3 \mathrm{~m}$ entre sí. En el tratamiento testigo se aplicó agua con pH de 6.5, misma que se empleó para preparar las soluciones acaricidas.

La efectividad biológica de los acaricidas se valoró mediante el conteo de los ácaros adultos que permanecieron vivos a los 2, 7, 14 y $21 \mathrm{~d}$ después de la aplicación de los tratamientos. Los datos de evaluación se obtuvieron de cuatro foliolos por planta, provenientes de cuatro plantas por tratamiento, mismos que se evaluaron y etiquetaron antes de la aplicación del acaricida. Se consideraron como ácaros vivos aquellos ejemplares que mostraron movimientos al perturbarse por $5 \mathrm{~s}$ con un pincel No. 000 (Helle y Overmeer 1985).

\section{Análisis estadístico}

Se realizó un análisis de varianza por fecha de evaluación. El número de ácaros adultos vivos encontrados a los 2, 7, 14 y 21 d después de la aplicación de los tratamientos, se transformó a porcentajes con respecto al valor inicial de ácaros registrados antes de la aplicación. Previo al análisis de varianza, los porcentajes se convirtieron a valores arcoseno $y_{i j}^{*}=\arcsin \sqrt{y_{i j} / 100}$ (Sokal y Rohlf 2012). La comparación de medias se realizó con la prueba de Tukey $(p=0.05)$. En ambos análisis se utilizó el programa estadístico $S A S{ }^{\circledR}$ versión 9.2 (SAS 2009).

\section{RESULTADOS Y DISCUSIÓN}

Previo a la aplicación de los acaricidas, la densidad promedio de ácaros vivos encontrados en las unidades experimentales fueron estadísticamente iguales entre sí $(p=0.18)$ (Tabla 2). Se observaron diferencias estadísticas entre acaricidas $(p<0.05)$, indicando la prueba de Tukey que cinco acaricidas proporcionaron los mejores porcentajes de control a los dos días de la aplicación, los cuales fueron bifentrina con $95.2 \%$ de efectividad, bifenazate con $87.5 \%$, acequinocyl con $86.6 \%$, spiromesifen con $83.7 \%$ y amitraz con $81.8 \%$. Además de estos productos químicos, los acaricidas fenpyroximate, clorfenapir, azufre, clohidrato de formetanato, milbemectina, propargite y fenazaquin tuvieron una efectividad de control del ácaro entre el 95 y $100 \%$ a los siete dias después de la aplicación. Mientras que 
Tabla 1. Características de los acaricidas comerciales evaluados contra Raoiella indica en Tabasco, México.

\begin{tabular}{|c|c|c|c|c|}
\hline $\begin{array}{l}\text { Ingrediente } \\
\text { activo }\end{array}$ & Nombre comercial & Grupo toxicológico $^{\dagger}$ & Modo de acción ${ }^{\dagger}$ & $\begin{array}{l}\text { Dosis } \boldsymbol{I}^{-1} \\
\text { de agua }\end{array}$ \\
\hline $\begin{array}{l}\text { Clorhidrato de } \\
\text { formetanato }\end{array}$ & Dicarzol $\mathbb{R}) 50$ PS & Grupo 1A. Carbamatos & Inhibidor de la acetilcolinesterasa & $1 \mathrm{~g}$ \\
\hline Bifentrina & Talstar ${ }^{\circledR} 100 \mathrm{CE}$ & Grupo 3A. Piretroides & Modulador del canal de sodio & $0.4 \mathrm{ml}$ \\
\hline Abamectina & Abamectina ${ }^{\circledR} \quad 1.8 \% \mathrm{CE}$ & $\begin{array}{l}\text { Grupo } 6 . \quad \text { Avermectinas, } \\
\text { milbemicinas }\end{array}$ & Activador del canal de cloro & $1 \mathrm{ml}$ \\
\hline Milbemectina & Koromite ${ }^{\circledR} 1 \%$ CE & $\begin{array}{l}\text { Grupo } 6 . \quad \text { Avermectinas, } \\
\text { milbemicinas }\end{array}$ & Activador del canal de cloro & $1 \mathrm{ml}$ \\
\hline Clofentezine & Acaristop ${ }^{R} 50 \mathrm{SC}$ & $\begin{array}{l}\text { Grupo } 10 \mathrm{~A} \text {. Clofentezin, } \\
\text { hexitiazox }\end{array}$ & Inhibidor del crecimiento de ácaros & $0.4 \mathrm{ml}$ \\
\hline Hexitiazox & Savey ${ }^{\circledR} 50 \mathrm{PH}$ & $\begin{array}{l}\text { Grupo } 10 \mathrm{~A} \text {. Clofentezin, } \\
\text { hexitiazox }\end{array}$ & Inhibidor del crecimiento de ácaros & $0.2 \mathrm{~g}$ \\
\hline Propargite & Omite ${ }^{\circledR} 6 \mathrm{E}$ & Grupo $12 \mathrm{C}$. Propargite & Inhibidor de la ATP sintasa mitocondrial & $2 \mathrm{ml}$ \\
\hline Clorfenapir & Sunfire ${ }^{\circledR} 2 \mathrm{CS}$ & $\begin{array}{l}\text { Grupo 13. Pirroles, dinitro- } \\
\text { fenoles, sulfluramida }\end{array}$ & $\begin{array}{l}\text { Desacoplador de la fosforilación oxida- } \\
\text { tiva vía disrupción del gradiente de pro- } \\
\text { tones }\end{array}$ & $1 \mathrm{ml}$ \\
\hline Flufenoxuron & Cascade ${ }^{\circledR} 100 \mathrm{CD}$ & Grupo 15. Benzoilureas & $\begin{array}{l}\text { Inhibidor de la biosíntesis de la quitina, } \\
\text { tipo } 0\end{array}$ & $0.7 \mathrm{ml}$ \\
\hline Amitraz & $\operatorname{Mitac}^{\circledR} 20 \mathrm{CE}$ & Grupo 19. Amitraz & Agonista de la octopamina & $1.5 \mathrm{ml}$ \\
\hline Acequinocyl & Kanemite ${ }^{\circledR} 15$ SC & Grupo 20B. Acequinocyl & $\begin{array}{l}\text { Inhibidor del transporte de electrones en } \\
\text { el complejo mitocondrial III }\end{array}$ & $1 \mathrm{ml}$ \\
\hline Fenazaquin & Magister $200^{\circledR}$ SC & $\begin{array}{l}\text { Grupo } 21 \mathrm{~A} \text {. Acaricidas e in- } \\
\text { secticidas METI }\end{array}$ & $\begin{array}{l}\text { Inhibidor del transporte de electrones en } \\
\text { el complejo mitocondrial I }\end{array}$ & $1.5 \mathrm{ml}$ \\
\hline Fenpyroximate & Avolant ${ }^{\circledR}$ & $\begin{array}{l}\text { Grupo } 21 \mathrm{~A} \text {. Acaricidas e in- } \\
\text { secticidas METI }\end{array}$ & $\begin{array}{l}\text { Inhibidor del transporte de electrones en } \\
\text { el complejo mitocondrial I }\end{array}$ & $1 \mathrm{ml}$ \\
\hline Spirodiclofen & Envidor ${ }^{\circledR}$ & $\begin{array}{l}\text { Grupo } 23 \text {. Derivados de los } \\
\text { ácidos tetrónico y tetrámico }\end{array}$ & Inhibidor de la acetil CoA carboxilasa & $1 \mathrm{ml}$ \\
\hline Spiromesifen & Oberon ${ }^{\circledR}$ & $\begin{array}{l}\text { Grupo 23. Derivados de los } \\
\text { ácidos tetrónico y tetrámico }\end{array}$ & Inhibidor de la acetil CoA carboxilasa & $1 \mathrm{ml}$ \\
\hline Azufre & Sultron ${ }^{\circledR} 725$ & Inorgánicos & Desconocido o incierto & $2.5 \mathrm{ml}$ \\
\hline Dicofol§ & AK-20 $\mathbb{R}$ & Organoclorados $\phi$ & Modulador del canal de sodio ${ }^{\phi}$ & $2 \mathrm{ml}$ \\
\hline Azadiractina & Progranik ${ }^{\circledR}$ Nimicide 80 & Desconocido & Desconocido o incierto & $2 \mathrm{ml}$ \\
\hline Bifenazate & Acramite ${ }^{\circledR} 50$ WS & Desconocido & Desconocido o incierto & $1.5 \mathrm{~g}$ \\
\hline
\end{tabular}

los productos de menor efectividad fueron spiridiclofen y hexitiazox. Cabe mencionar, que la sola aplicación de agua corriente (tratamiento testigo) afectó de forma negativa la presencia de $R$. indica.

En general, a los $21 \mathrm{~d}$ después de la aplicación, todos los productos acaricidas evaluados mostraron potencial para utilizarse en el control de $R$. indica, proporcionando opciones para un programa de control químico en plantaciones de cocotero. Los estudios previos sobre este tema son escasos, pero de acuerdo con Rodrigues y Peña (2012), los acaricidas acequinocyl, milbemectina y spiromesifen también son efectivos para reducir poblaciones de $R$. indica en plantaciones de cocotero; mientras que las aspersiones de abamectina, milbemectina y azufre fueron efectivos en plantaciones de cocotero y bananas. En bioensayos de laboratorio, con adultos de $R$. indica a la concentración letal al $90 \%\left(\mathrm{CL}_{90}\right)$ los acaricidas milbemectina, abamectina y fenpyroximate fueron más tóxicos que spiromesifen, clorfenapir y hexitiaxox (De Asiss et al. 2013). Bajo la misma metodología de evaluación, con adultos de $R$. indica en Tabasco, México, los acaricidas fenazaquim, milbemectina, abamectina y dicofol fueron más tóxicos que propargite, clorhidrato de formetanato, azufre y bifentrina 
Correa-Méndez et al.

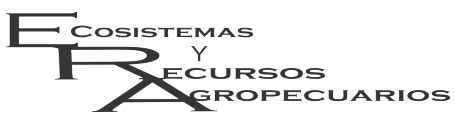

Control químico del ácaro rojo

Ecosist. Recur. Agropec.

5(14):319-326,2018

Tabla 2. Efectividad biológica de acaricidas en poblaciones de Raoiella indica en plantas de cocotero híbrido (Alto del Pacífico x Enano Malayo) en Tabasco, México.

\begin{tabular}{lrrrrr}
\hline \multirow{2}{*}{ Tratamiento } & \multicolumn{4}{c}{ Porcentaje promedio $( \pm \mathrm{EE})$ de ácaros adultos vivos por foliolo } \\
\cline { 2 - 6 } & \multicolumn{1}{c}{ 1 DAT } & \multicolumn{1}{c}{ D DDT } & \multicolumn{1}{c}{ 7 DDT } & $14 \mathrm{DDT}^{\S}$ & $21 \mathrm{DDT}^{\S}$ \\
\hline Bifentrina & $265.5 \pm 83.1$ & $4.8 \pm 2.7^{a}$ & $0.1 \pm 0.1^{a}$ & $0.2 \pm 0.2^{a}$ & $0^{a}$ \\
Acequinocyl & $213.5 \pm 64.1$ & $13.4 \pm 6.6^{a b}$ & $0^{a}$ & $0^{a}$ & $0.2 \pm 0.1^{a}$ \\
Bifenazate & $198.4 \pm 44.1$ & $12.5 \pm 2.2^{a b c}$ & $0.4 \pm 0.4^{a b}$ & $1.8 \pm 1.8^{a b}$ & $0.2 \pm 0.2^{a}$ \\
Spiromesifen & $170.1 \pm 40.3$ & $16.3 \pm 6.7^{a b c d}$ & $0.9 \pm 0.6^{a b}$ & $2.3 \pm 1.9^{a b c}$ & $0.6 \pm 0.3^{a}$ \\
Amitraz & $196.6 \pm 58.6$ & $18.2 \pm 7.4^{a b c d}$ & $0^{a}$ & $0^{a}$ & $0.1 \pm 0.1^{a}$ \\
Spirodiclofen & $166.3 \pm 37.4$ & $20.6 \pm 3.7^{a b c d}$ & $22.7 \pm 9.6^{c d}$ & $19.2 \pm 4.7^{a b c}$ & $11.7 \pm 4.9^{a}$ \\
Fenpyroximate & $197.4 \pm 65.1$ & $20.6 \pm 3.5^{a b c d}$ & $0^{a}$ & $0.3 \pm 0.2^{a}$ & $0.8 \pm 0.5^{a}$ \\
Clorfenapir & $264.2 \pm 98.6$ & $23.8 \pm 4.8^{a b c d}$ & $0^{a}$ & $0^{a}$ & $0^{a}$ \\
Azufre & $208.4 \pm 64.2$ & $25.1 \pm 5.3^{a b c d}$ & $1.2 \pm 1.2^{a b}$ & $3.7 \pm 1.9^{a b c}$ & $2.7 \pm 1.5^{a}$ \\
Clorhidrato de formentanato & $244.5 \pm 97.9$ & $25.2 \pm 5.1^{a b c d e}$ & $0.8 \pm 0.8^{a b}$ & $4.3 \pm 3.6^{a b c}$ & $0.5 \pm 0.5^{a}$ \\
Dicofol & $209.3 \pm 76.1$ & $28.5 \pm 5.1^{b c d e}$ & $5.9 \pm 2^{a b c}$ & $0.3 \pm 0.2^{a}$ & $0.3 \pm 0.2^{a}$ \\
Azadiractina & $187.9 \pm 57.6$ & $29.5 \pm 7.8^{b c d e}$ & $14.6 \pm 3.2^{c}$ & $5.9 \pm 2.8^{a b c}$ & $3.9 \pm 1.2^{a}$ \\
Clofentezine & $237.2 \pm 96.0$ & $32.2 \pm 8.4^{b c d e}$ & $5.7 \pm 2.2^{a b c}$ & $4.7 \pm 1.8^{a b c}$ & $4.7 \pm 0.9^{a}$ \\
Abamectina & $187.2 \pm 41.3$ & $34.1 \pm 6.9^{b c d e}$ & $6.2 \pm 2.1^{a b c}$ & $4.1 \pm 1^{a b c}$ & $0.7 \pm 0.5^{a}$ \\
Milbemectina & $209.7 \pm 83.2$ & $35.3 \pm 10.8^{b c d e}$ & $1 \pm 1^{a b}$ & $11.7 \pm 9^{a b c}$ & $5.6 \pm 3.7^{a}$ \\
Hexitiazox & $177.3 \pm 41.3$ & $36.9 \pm 9.6^{b c d e}$ & $21.7 \pm 8.1^{c d}$ & $18.4 \pm 5.6^{b c}$ & $10.8 \pm 6.5^{a}$ \\
Flufenoxuron & $182.9 \pm 38.0$ & $39.6 \pm 9.1^{c d e}$ & $9.3 \pm 3.0^{b c}$ & $4.2 \pm 1.6^{a b c}$ & $5.6 \pm 2.2^{a}$ \\
Propargite & $283.8 \pm 98.1$ & $42.2 \pm 15.5^{d e}$ & $0^{a}$ & $0^{a}$ & $0^{a}$ \\
Fenazaquin & $230.9 \pm 88.3$ & $54.3 \pm 13^{e}$ & $0^{a}$ & $0^{a}$ & $0^{a}$ \\
Testigo (agua) & $245.5 \pm 98.4$ & $43.1 \pm 11.2^{d e}$ & $41.5 \pm 13.6^{d}$ & $71.0 \pm 14.5^{d}$ & $95.2 \pm 16.6^{b}$ \\
\hline
\end{tabular}

${ }^{\dagger}$ Basado en un área foliar de $70 \mathrm{~cm}^{2}$. DAT días antes del tratamiento. DDT días después del tratamiento. " Promedios estadísticamente iguales con respecto al testigo $(\mathrm{p}=0.18)$. §Promedios seguidos por letras diferentes son significativamente diferentes (Tukey, $\mathrm{p} \leq 0.05$ ). Los datos originales fueron transformados a $y_{i j}^{*}=\arcsin \sqrt{y_{i j} / 100}$.

(Sánchez-Vázquez et al. 2017). En general, los acaricidas que comparativamente fueron más tóxicos para los adultos de $R$. indica en condiciones de laboratorio ( $\left.\mathrm{CL}_{90}\right)$ (Sánchez-Vázquez et al. 2017), no fueron los más efectivos en campo. Lo anterior, se puede deber a que la efectividad biológica en condiciones de campo no corresponde al comportamiento toxicológico observado bajo condiciones de laboratorio, ya que la determinación de laboratorio no considera las pérdidas por arrastre, fotodescomposición, termorregulación, escape del organismo, entre otros factores (Lagunes-Tejeda et al. 2009). En México, el uso de los acaricidas abamectina, azufre y spiridiclofen están autorizados de forma temporal para su uso en la campaña fitosanitaria contra $R$. indica (SENASICA 2015), los cuales mostraron una efectividad del 94,99 y $77 \%$, respectivamente, a los $7 \mathrm{~d}$ después del tratamiento.

El control químico de ácaros fitófagos es problemático, debido al alto potencial de desarrollo de resistencia (Whalon et al. 2008). En particular, el ácaro rojo $R$. indica tiene un ciclo de desarrollo corto y fecundidad alta con reproducción sexual y asexual por partenogénesis arrenotóquica (Nageshachandra y Channabasavanna 1984, González y Ramos 2010); características biológicas que pueden acelerar el desarrollo de la resistencia en un corto plazo (Whalon et al. 2008). De acuerdo a Sawicki y Denholm (1987), el manejo de la resistencia a los plaguicidas es un conjunto de estrategias con la premisa básica de conservar la susceptibilidad a los plaguicidas, mediante uso racional y la restricción de tratamientos para prevenir la selección de individuos resistentes $\mathrm{y}$, de este modo, prolongar la vida útil de los productos químicos. Por lo tanto, considerando los 12 productos acaricidas con mejor desempeño para el control químico de $R$. indica y de acuerdo con el orden de los grupos toxicológicos establecido por el IRAC (Sparks y Nauen 2015), se propone utilizar la secuencia siguiente: 1) clorhidrato de formetanato, 2) bifentrina, 3) milbemectina, 4) propargite, 5) clorfenapir, 6) amitraz, 7) acequinocyl, 8) bifenazate, 9) fenazaquin o fenpyroximate, 10) spiromesifen y 11) azufre elemental. En todo caso, es importante realizar una rotación o alternancia del uso de estos 
acaricidas para el control químico de $R$. indica que permitirá prolongar la vida útil de estos productos (Whalon et al. 2008). En conclusión, los acaricidas bifentrina, acequinocyl, bifenazate, spiromesifen, amitraz, fenpyroximate, clorfenapir, azufre, clorhidrato de formetanato, milbemectina, propargite y fenazaquin fueron los más efectivos, al reducir más de $95 \%$ de las poblaciones de $R$. indica a los siete días después de la aplicación.

\section{AGRADECIMIENTOS}

A la Universidad Juárez Autónoma de Tabasco por el financiamiento del proyecto: Potencial de daño del ácaro rojo Raoiella indica en cultivares de plátano y efectividad de acaricidas para su control, Clave: UJAT-2013-IB-26.

\section{LITERATURA CITADA}

Carrillo D, Amalin D, Hoisen F, Roda A, Duncan RE, Peña EJ (2012) Host plant range of Raoiella indica (Acari: Tenuipalpidae) in areas of invasion of the New World. Experimental and Applied Acarology 57: 271-289.

COFEPRIS (2015) Búsqueda de registros de: plaguicidas y nutrientes vegetales. Secretaría de Salud. Comisión Federal para la Protección contra Riesgos Sanitarios. México. http://189.254.115.250/Resoluciones/Consultas/ConWebRegPlaguicida.asp. Fecha de consulta 10 de agosto de 2015.

De Assis CPO, De Morais EGF, Gondim MGC (2013) Toxicity of acaricides to Raoiella indica and their selectivity for its predator, Amblyseius largoensis (Acari: Tenuipalpidae: Phytoseiidae). Experimental and Applied Acarology 60: 357-365.

Dekeyser MA (2005) Review acaricide mode of action. Pest Management Science 61: 103-110.

Flechtmann CHW, Etienne J (2004) The red palm mite, Raoiella indica Hirst, a threat to palms in the Americas (Acari: Prostigmata: Tenuipalpidae). Systematic and Applied Acarology 9: 109-110

Fountain MT, Harris AL, Cross JV (2010) The use of surfactants to enhance acaricide control of Phytonemus pallidus (Acari: Tarsonemidae) in strawberry. Crop Protection 29: 1286-1292.

González RAI, Ramos M (2010) Desarrollo y reproducción de Raoiella indica Hirst. (Acari: Tenuipalpidae) en laboratorio. Revista Protección Vegetal 25: 7-10.

Helle W, Overmeer JPW (1985) Toxicological test methods. In: Helle W, Sabelis MW (eds) Spider Mites: their biology, natural enemies and control. Elsevier Science Publishing Company. Inc. USA. pp: 391395.

Kane EC, Ochoa R, Mathurin G, Erbe EF, Beard JJ (2012) Raoiella indica (Acari: Tenuipalpidae): an exploding mite pest en the neotropics. Experimental and Applied Acarology 57: 215-225.

Lagunes-Tejeda A, Rodríguez-Maciel JC, De Loera-Barocio JC (2009) Susceptibilidad a insecticidas en poblaciones de artrópodos de México. Agrociencia 43: 173-196.

Larios-Romero J, Hernández J (1987) Condiciones ambientales para la agricultura en el estado de Tabasco. Revista de Geografía Agrícola 1314: 33-42.

Marcic D (2012) Acaricides in modern management of plant-feeding mites. Journal of Pest Science 85: 395-408.

Nageshachandra BK, Channabasavanna GP (1984) Development and ecology of Raoiella indica Hirst (Acari: Tenuipalpidae) on coconut. In: Griffiths DA, Bowman CE (eds). Acarology VI. Vol. 2. John Wiley \& Sons, NY, USA. pp: 785-798. 
NAPPO (2009) Detección del ácaro rojo de las palmas (Raoiella indica) en Isla Mujeres y Cancún, Quintana Roo, México. Sistema de Alerta Fitosanitaria-NAPPO. Organización Norteamericana de Protección a las Plantas. http://www.pestalert.org/espanol/oprDetail.cfm?oprID=406. Fecha de consulta 14 de agosto de 2017.

Polanco-Arjona CA, Osorio-Osorio R, Hernández-Hernández LU, Márquez-Quiroz C, De la Cruz-Lázaro E, Salinas-Hernández RM, Hernández-García V (2017) Colonization, Abundance, and Damage of Raoiella indica Hirst on Cultivars of Musa spp. at Tabasco, México. Southwestern Entomologist 42: 363-374.

Roda A, Nachman G, Hosein F, Rodrigues JCV, Peña EJ (2012) Spatial distributions of the red palm mite, Raoiella indica (Acari: Tenuipalpidae) on coconut and their implications for development of efficient sampling plans. Experimental and Applied Acarology 57: 291-308.

Rodrigues JCV, Ochoa R, Kane EC (2007) First report of Raoiella indica Hirst (Acari: Tenuipalpidae) and its damage to coconut palms in Puerto Rico and Culebra Island. International Journal Acarology 33: 3-5.

Rodrigues JCV, Peña EJ (2012) Chemical control of the red palm mite Raoiella indica (Acari: Tenuipalpidae) in banana and coconut. Experimental and Applied Acarology 57: 317-329.

Sánchez-Vázquez E, Osorio-Osorio R, Hernández-Hernández LU, Hernández-García V, Márquez-Quiroz C, De la Cruz-Lázaro E (2017) Toxicidad de acaricidas para el ácaro rojo de las palmas Raoiella indica (Acari: Tenuipalpidae). Agrociencia 51: 81-90.

SAS (2009) SAS/STAT 9.2 User's Guide. Second Edition Cary, NC, USA. 7869p.

Sawicki RM, Denholm L (1987) Management of resistance to pesticides in cotton pests. Tropical Pest Management 33: 262-272.

SENASICA (2015) Acaro rojo de las palmas (Raoiella indica Hirst). Ficha técnica No. 14. Servicio Nacional de Sanidad, Inocuidad y Calidad Agroalimentaria-Dirección General de Sanidad Vegetal, Programa de Vigilancia Epidemiológica Fitosanitaria. 22p. http://www.gob.mx/cms/uploads/attachment/file/120233/Ficha_Acaro_rojo_de_las_palmas_2015.pdf. Fecha de consulta 9 de enero de 2017.

SENASICA (2017) Acaro rojo de las palmas (Raoiella indica Hirst). Campañas y programas fitosanitarios. Servicio Nacional de Sanidad, Inocuidad y Calidad Agroalimentaria, Dirección General de Sanidad Vegetal, Programa de Vigilancia Epidemiológica Fitosanitaria. https://www.gob.mx/senasica/documentos/ acaro-rojo-de-las-palmas-110890. Fecha de consulta 14 de agosto de 2017.

Sokal, RR and FJ Rohlf (2012) Biometry. Fourth Edition. W.H. Freeman and Co. New York, USA. 937p.

Sparks TC, Nauen R (2015) IRAC: Mode of action classification and insecticide resistance management. Pesticide Biochemistry and Physiology 121: 122-128.

Van Leeuwen T, Tirry L, Yamamoto A, Nauen R, Dermauw W (2015) The economic importance of acaricides in the control of phytophagous mites and an update on recent acaricide mode of action research. Pesticide Biochemistry and Physiology 121: 12-21.

Van Leeuwen T, Vontas J, Tsagkarakou A, Dermauw W, Tirry L (2010) Acaricide resistance mechanisms in the two-spotted spider mite Tetranychus urticae and other important Acari: A review. Insect Biochemistry and Molecular Biology 40: 563-572

Whalon ME, Mota-Sánchez D, Hollingworth RM (2008) Analysis of Global Pesticide Resistance in Arthropods. In: Whalon ME, Mota-Sánchez D, Hollingworth RM (eds). Global Pesticide Resistance in Arthropods. CAB International. UK. pp: 5-31. 
Correa-Méndez et al.

Ecosistemas

Pecursos

Mecursos
Control químico del ácaro rojo

Ecosist. Recur. Agropec.

5(14):319-326,2018

Whalon ME, Mota-Sánchez D, Hollingworth RM (2016) Arthropod Pesticide Resistance Database. http://www.pesticideresistance.org/index.php. Fecha de consulta 27 de enero de 2016. 